Letter

\title{
Terahertz Gas-Phase Spectroscopy Using a Sub-Wavelength Thick Ultrahigh-Q Microresonator
}

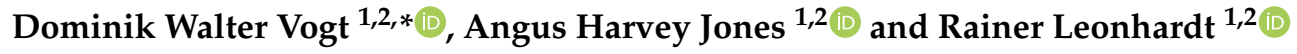 \\ 1 Department of Physics, The University of Auckland, Auckland 1010, New Zealand; \\ ajon083@aucklanduni.ac.nz (A.H.J.); r.leonhardt@auckland.ac.nz (R.L.) \\ 2 The Dodd-Walls Centre for Photonic and Quantum Technologies, Dunedin 9016, New Zealand \\ * Correspondence: d.vogt@auckland.ac.nz
}

Received: 28 April 2020; Accepted: 22 May 2020; Published: 25 May 2020

\begin{abstract}
The terahertz spectrum provides tremendous opportunities for broadband gas-phase spectroscopy, as numerous molecules exhibit strong fundamental resonances in the $\mathrm{THz}$ frequency range. However, cutting-edge $\mathrm{THz}$ gas-phase spectrometer require cumbersome multi-pass gas cells to reach sufficient sensitivity for trace level gas detection. Here, we report on the first demonstration of a $\mathrm{THz}$ gas-phase spectrometer using a sub-wavelength thick ultrahigh-Q $\mathrm{THz}$ disc microresonator. Leveraging the microresonator's ultrahigh quality factor in excess of 120,000 as well as the intrinsically large evanescent field, allows for the implementation of a very compact spectrometer without the need for complex multi-pass gas cells. Water vapour concentrations as low as 4 parts per million at atmospheric conditions have been readily detected in proof-of-concept experiments.
\end{abstract}

Keywords: terahertz spectroscopy; microresonator; water vapour sensing

\section{Introduction}

Terahertz (THz) radiation, located between microwave and infrared frequencies, bridges the gap between electronics and optics, and has attracted significant interest for broadband gas-phase spectroscopy. In particular, because of the abundance of strong fundamental absorption lines of a large variety of molecules in this frequency range [1-9]. However, current cutting edge $\mathrm{THz}$ gas-phase spectrometers still require cumbersome multi-pass gas cells to reach sufficient sensitivity for trace level gas detection [10-13]. In order to fully exploit the potential of $\mathrm{THz}$ gas-phase spectroscopy, novel sensors that are highly frequency selective and sensitive to changes in the surrounding medium are required. Here, we present the first demonstration of $\mathrm{THz}$ gas-phase spectroscopy by exploiting the unprecedented frequency selectivity and environmental sensitivity characteristic to sub-wavelengths thick ultrahigh quality factor (ultrahigh-Q) disc microresonators. The specifically designed ultrahigh-Q THz disc microresonators allow for the implementation of compact, highly sensitive $\mathrm{THz}$ gas-phase spectrometers.

Ultrahigh-Q microresonators are devices that can confine radiation with exquisitely low losses. Such low losses give rise to ultra-narrow resonance features that imply very fine frequency selectivity [14]. Moreover, even a slight change in the surrounding medium will perturb the resonances to a measurable extent, allowing for environmental sensitivity. To achieve sufficiently low losses (high Q-factors), the resonators must be formed from a single piece of material. While such monolithic resonators have been extensively studied at optical frequencies [15-22], they remain largely unexplored in the $\mathrm{THz}$ domain. It is only very recently that this concept has been translated into the $\mathrm{THz}$ domain [23-31], achieving unprecedented Q-factors more than two orders of magnitude higher compared to previous systems [29]. Unfortunately, while the demonstrated devices highlight the potential of monolithic $\mathrm{THz}$ resonators, their characteristics are still insufficient for demanding gas-phase spectroscopy applications. This is because in traditional resonator designs (e.g., a sphere, 
disc, or ring), the $\mathrm{THz}$ radiation resides predominantly inside the resonator, limiting the devices' Q-factor and environmental sensitivity. We overcome this deficiency by exploiting the sub-wavelength confinement offered by a novel class of thin-disk microresonators, where the radiation is forced to reside outside of the resonator, as a large evanescent field (see Figure 1a,b). As a proof of concept, we demonstrate as an exemplar the detection of less than 4 parts-per-million by volume (ppmv) of water vapour at ambient conditions. Novel sensing schemes for minute water vapour concentrations are in immediate interest, for example, for lithium-ion battery manufacturing, semiconductor fabrication and pharmaceutical industries [11]. However, we envision that the presented compact THz gas-phase spectrometer with the ultrahigh-Q THz disc microresonator will enable a myriad of possibilities in, for example, medical or industrial applications.
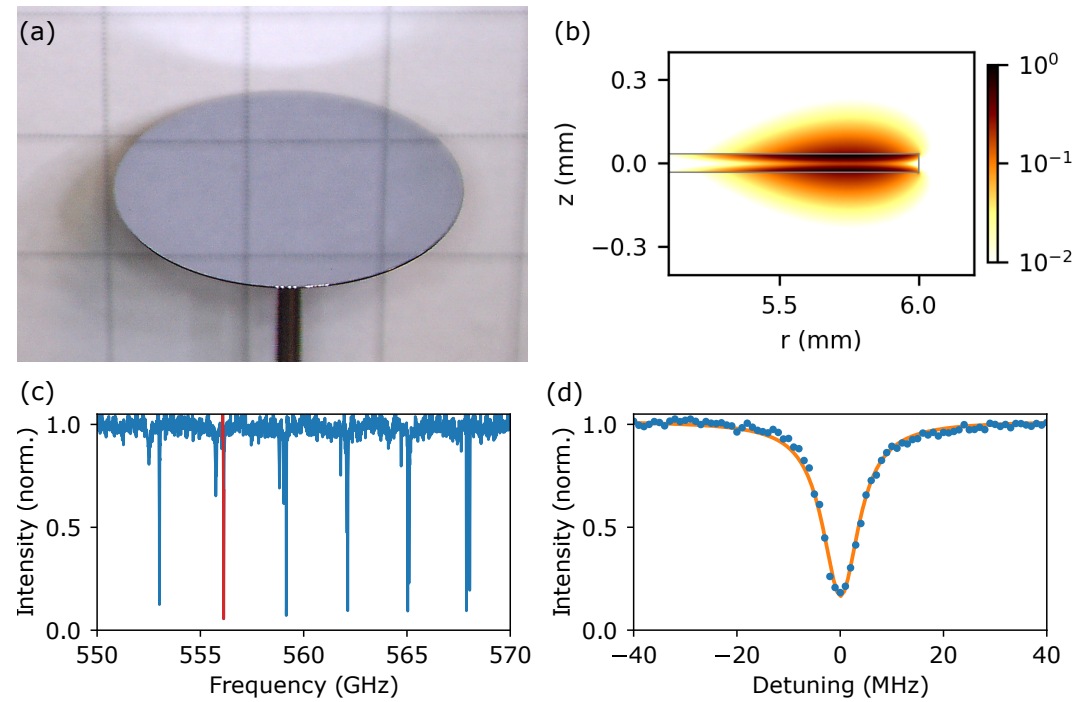

Figure 1. (a) Microscope image of a $12 \mathrm{~mm}$ diameter, $66 \pm 1 \mu \mathrm{m}$ thick HRFZ-Si THz disc microresonator. The resonator is mounted on a $1 \mathrm{~mm}$ diameter aluminium rod. (b) Corresponding simulated intensity distribution (normalised) on logarithmic scale showing the large extend of the evanescent field. The microresonator cross-section is indicated with grey lines. Please note that all simulations presented in this work are performed with COMSOL Multiphysics ${ }^{\circledR}$ software [32], and fabrication imperfections are not considered in the simulations. (c) Measured intensity profile of the $\mathrm{THz}$ disc microresonator showing the fundamental mode. (d) Resonance at $0.5561 \mathrm{THz}$ (highlighted in red in sub-figure (c)) close to critical coupling. The frequency step size is $1 \mathrm{MHz}$ (blue dots). The fitted analytical model [33] is shown with the orange solid line.

\section{Materials and Methods}

Key to the $\mathrm{THz}$ disc microresonator's unprecedented frequency selectivity and environmental sensitivity, apart from a low-loss substrate (here, high resistivity float-zone grown silicon), is the sub-wavelength thickness of the disc resonator. Thinning the disc to a fraction of the free-space wavelength shifts a large proportion of the $\mathrm{THz}$ radiation - that would otherwise be confined to the lossy resonator substrate- to the surrounding environment. This not only reduces the impact of the disc's material absorption (increasing the Q-factor); but also intrinsically produces a large evanescent field, leading to a significant overlap with the resonator's environment. Figure 1a,b show images of a fabricated sub-wavelength thick $\mathrm{THz}$ disc microresonator and the corresponding simulated intensity distribution. The fabricated microresonator has a $12 \mathrm{~mm}$ diameter and a thickness of $66 \pm 1 \mu \mathrm{m}$, corresponding to $\lambda_{0} / 8$ at $0.6 \mathrm{THz}$. The calculated evanescent field fraction $f$ (ratio of the energy of the electromagnetic radiation outside of the resonator to the overall energy) of this disc is $52 \%$. This compares to a calculated evanescent field fraction of about $0.3 \%$ for a $4 \mathrm{~mm}$ diameter spherical silicon $\mathrm{THz}$ microresonator, highlighting the enhancement in evanescent field of the sub-wavelength thick disc compared to a spherical resonator design. 
As mentioned above, a low-loss resonator substrate is essential as even with a thickness of $\lambda_{0} / 8$ about $50 \%$ of the total energy remain in the disc's substrate. Here, the disc microresonator is made of high resistivity float-zone grown silicon (HRFZ-Si) with a resisitivity of $>10 \mathrm{k} \Omega \mathrm{cm}$, as this is one of the lowest loss materials known in the THz domain [29]. Fabrication of the discs is achieved by thinning a standard $100 \mu \mathrm{m}$ thick, 2 inch diameter wafer and subsequent laser micro-machining. Finally, the disc's rim is polished using a fine diamond slurry to remove any imperfections from the laser micro-machining. The disc microresonator's diameter and thickness are chosen to maximise the Q-factor and extent of the evanescent field at a specific design frequency-here about $0.6 \mathrm{THz}$ according to the water vapour absorption line at $0.557 \mathrm{THz}$ [34]. The detailed intricacies of the resonator design are outlined elsewhere [35].

The fundamental resonances of the disc microresonator are shown for the frequency range from $0.55 \mathrm{THz}$ to $0.57 \mathrm{THz}$ in Figure $1 \mathrm{c}$ as an example. The free spectral range (FSR) of the fundamental mode is about $3 \mathrm{GHz}$. Figure $1 \mathrm{~d}$ shows the resonance at $0.556 \mathrm{THz}$, with an intrinsic (no coupling, in an ideal non-absorbing gas) Q-factor of about 120,000. Experimentally, the intrinsic Q-factor is obtained by fitting the measured $Q$ vs ppmv dependence with an analytical model [33] and extrapolating to 0 ppmv (see detailed discussion below).

The resonances shown in Figure $1 \mathrm{c}$ are excited using evanescent coupling with a single-mode air-flouropolymer-silica step-index waveguide with a $200 \mu \mathrm{m}$ silica core that is coated with a flouropolymer layer of $12.5 \mu \mathrm{m}$ thickness. The waveguide provides single-mode operation in the frequency range from $0.4 \mathrm{THz}$ to $0.65 \mathrm{THz}$, with an effective refractive index of 1.27 to 1.37 from $540 \mathrm{GHz}$ to $600 \mathrm{GHz}$. The coupling strength of the resonance can be tuned by changing the distances between the waveguide and the microresonator. A schematic of the experimental setup is shown in Figure 2. The THz radiation is generated and detected using fiber-coupled photoconductive antennas (PCA) with a continuous-wave (CW) THz system from Toptica (TeraScan 1550 [36]). The smallest step-size of the CW-THz system is about $1 \mathrm{MHz}$, which is essential in sufficiently resolving the very narrow ultrahigh-Q THz resonances with a typical line width of less than $10 \mathrm{MHz}$. The data analysis is performed using a Hilbert transform, which is explained in great detail elsewhere [37,38]. The intensity profiles shown in Figure 1c,d are obtained by normalising a sample scan (waveguide transmission with the coupled resonator) with a reference scan (waveguide transmission without the resonator). This eliminates undesired effects, like standing waves in the setup and frequency-dependent performance of the $\mathrm{THz}$ antennas, thus solely provides the frequency response of the $\mathrm{THz}$ microresonator.

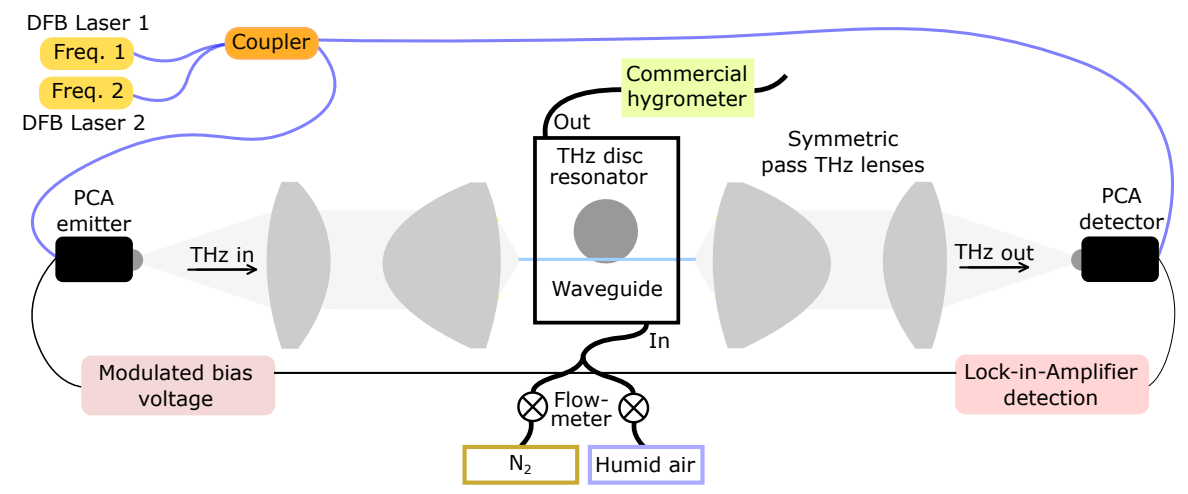

Figure 2. Schematic of the gas-phase $\mathrm{THz}$ spectrometer with a commercial CW-THz system and a sub-wavelenth thick $\mathrm{THz}$ disc microresonator. The $\mathrm{THz}$ microresonator is mounted on a 3D translation stage to control the position of the resonator relative to the air-flouropolymer-silica waveguide. Both the horizontal and vertical position of the waveguide relative to the resonator were monitored with digital microscopes. Because of the intriguing field distribution, best coupling is achieved by placing the waveguide above or below the edge of the disc. Strong coupling is typically achieved at a position of the waveguide of about $200 \mu \mathrm{m}$ inside from the edge of the microresonator and a gap of about $100 \mu \mathrm{m}-200 \mu \mathrm{m}$ to the microresonator. The deployed symmetric-pass $\mathrm{THz}$ lenses are specifically designed to achieve high coupling efficiency to the sub-wavelength waveguide [39]. 
The operational principle for the gas-phase spectroscopy using the $\mathrm{THz}$ disc microresonator is to monitor a change in Q-factor under variation of the gas concentration in the resonator's environment. This modality is known to be more sensitive than, for example, observing resonance frequency shifts [40], and takes advantage of the strong gas absorption lines in the THz domain. Of course, this requires the microresonator's resonances to coincide with the desired gas absorption lines. The spectral position of the resonances is determined by the HRFZ-Si resonator's geometry and can subsequently be tuned, for example, by using thermal mechanisms [41,42]. Here, the $12 \mathrm{~mm}$ diameter disc shown in Figure 1a has an ultrahigh-Q resonance at $0.5561 \mathrm{THz}$ at room temperature which is sufficiently close to the water vapour absorption line at $0.5569 \mathrm{THz}$ (with a line width of about $6 \mathrm{GHz}$ ), and therefore no tuning was performed.

The location of the gas-cell used in this proof-of-concept experiment is indicated in Figure 2. The gas-cell is milled from Perspex and features a rectangular design with two Teflon windows to ensure a high transmission of the THz signal. The windows are also used as mounts for the waveguide with the end-faces of the waveguide protruding to the outside of the gas cell. The gas-cell is designed to operate under a constant gas flow with the inlet and the outlet at opposite sides of the gas-cell. The humidity level is adjusted by controlling the flows of Nitrogen and humid air at the inlet of the gas cell, and monitored at the output using a high-end commercial hygrometer (Vaisala DMT152) which serves as a reference for the actual water vapour concentration. We note, that this proof-of-concept experimental setup is not suitable for field work, however, we envision, that in the future a more integrated approach will lead to a much lower susceptibility to vibrations. The experimental procedure is as follows: (1) Adjustment of the desired gas concentration and ensuring a constant gas flow/ppmv reading. (2) Performing a reference scan, that is, the waveguide transmission without coupling to the $\mathrm{THz}$ disc microresonator. (3) Adjust the distance between the waveguide and the resonator to achieve strong coupling and perform a sample scan. (4) Finally, the intensity and phase profiles obtained from the waveguide transmission are fitted simultaneously with the complex analytical model to obtain the resonator's Q-factor at this specific gas concentration. Utilising intensity and phase information provides a higher confidence in the obtained Q-factor than solely relying on the intensity. Finally, steps (2) to (4) are repeated several times to further reduce the standard error in Q-factor.

\section{Results and Discussion}

Figure 3 shows the measured intensity and phase profiles (blue dots) of the resonance at $0.5561 \mathrm{THz}$ at (a) $7 \mathrm{ppmv}$ and (b) $120 \mathrm{ppmv}$ water vapour concentrations. The fitted analytical model (orange solid lines) is in very good agreement with the measurements. Comparison of Figure 1a,b shows the broadening (lower Q-factor) of the investigated resonances due to the increased water vapour absorption, and nicely demonstrates the principle of the sensing modality.

The measured Q-factors (blue dots) for a range of water vapour concentrations from $4 \mathrm{ppmv}$ to $120 \mathrm{ppmv}$ (0.01-0.49\% relative humidity) are summarised in Figure 4 . The horizontal error-bars show the uncertainty in the ppmv reading as calculated from the specified $\pm 2{ }^{\circ} \mathrm{C}$ uncertainty in the frost point temperature displayed by the commercial hygrometer. An uncertainty of $\pm 2{ }^{\circ} \mathrm{C}$ in frost point temperature converts to about $20 \%$ to $30 \%$ uncertainty in the ppmv reading in the range from 4 to $120 \mathrm{ppmv}$. This large uncertainty is typical for a high-end commercial hygrometer in this concentration range. While this somewhat limits the ability to calibrate the presented $\mathrm{THz}$ spectrometer with sub-wavelength thick $\mathrm{THz}$ disc microresonator, it also reveals the persisting challenges in the field of low water vapour concentration detection, and the need for better technologies. The vertical error-bars result from the uncertainty in the Q-factor extracted from the analytical model fitted to the measurements. A main limiting factor in the uncertainty of the fitted Q-factor is the frequency resolution and stability of the deployed CW-THz System. A higher frequency resolution/stability, as for example with a comb-locked frequency-domain terahertz spectrometer [43], would greatly reduce the uncertainty, and ultimately improve the capability to distinguish small changes in the Q-factor. 

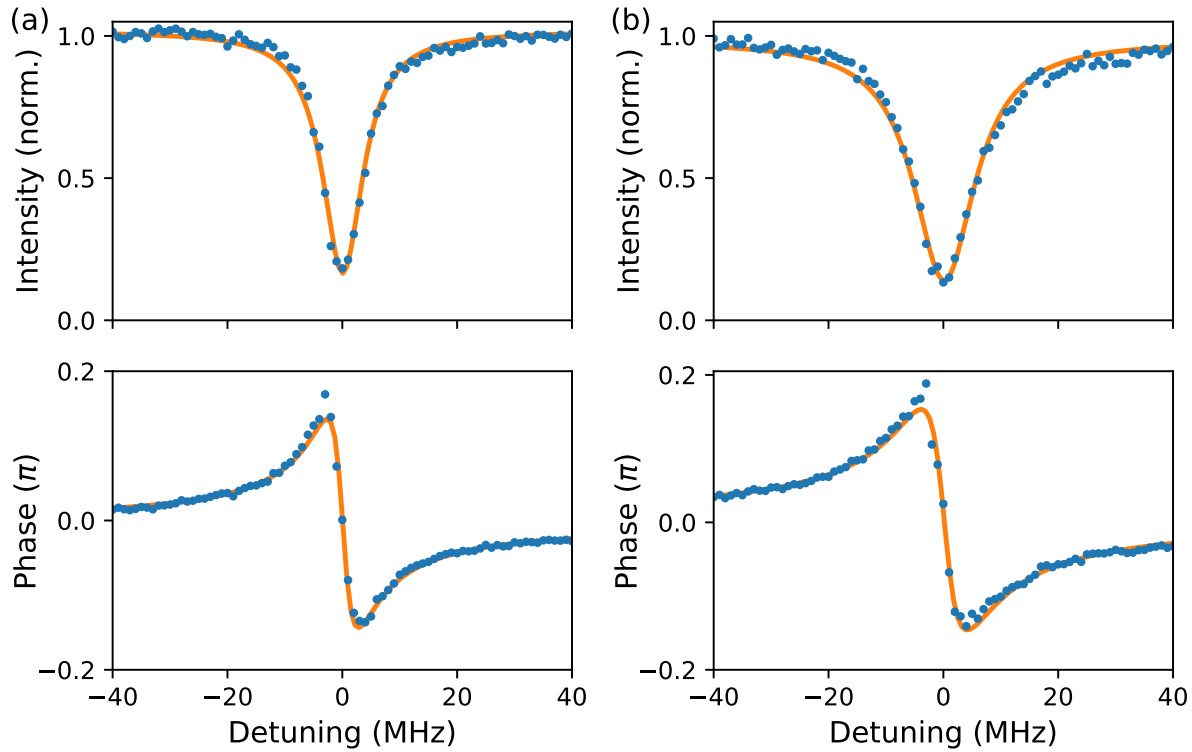

Figure 3. (a) Measured intensity and phase profiles (blue dots) of the resonance at $0.5561 \mathrm{THz}$ at $7 \mathrm{ppmv}$ with the corresponding fit (orange solid lines). (b) The same resonance at 120 ppmv water vapour concentration. Both measurements are recorded with similar coupling strength to ease comparison.

The change in Q-factor as a function of the water vapour concentration can be modelled by the following analytic equation:

$$
Q=\frac{C}{\alpha_{i}+f \alpha_{\text {gas }}\left(\nu_{0}, p p m v\right)} .
$$

With $\alpha_{i}$ the effective resonator loss (includes material loss, radiation loss, scattering loss etc.) surrounded by a lossless gas, and $\alpha_{\text {gas }}\left(v_{0}, p p m v\right)$ the gas absorption at the resonator resonance frequency $v_{0}$ as a function of water vapour concentration. $C$ is a constant defined as $C=2 \pi \mathrm{n}_{\mathrm{s}} v_{0} / \mathrm{c}$, with $n_{s}$ the refractive index of the resonator substrate $\left(n_{\mathrm{HRFZ}-\mathrm{Si}}=3.416\right.$ at $\left.0.6 \mathrm{THz}\right)$, and $\mathrm{c}$ the speed of light [33]. This functional dependence has been used in Figure 4 to fit the solid orange curve to our data points, and the trend of the data agrees very well with the analytic expression. For a more quantitative comparison $\alpha_{g a s}(\nu, p p m v)$ can be expressed using the HITRAN-database notation as follows [34]:

$$
\alpha_{\text {gas }}(v, p p m v)=N S g(v) p .
$$

With $N$ the total number of molecules of absorbing gas, $\mathrm{S}$ is the molecular line intensity, $g(v)$ the absorption line profile and $p$ the gas partial pressure, which can be related to the $p p m v$ via $p p m v=p / p_{\text {atm }} 10^{6}$, with $p_{\text {atm }}$ the total pressure in the gas cell (here typically 1 atmosphere). For the absorption line profile $g(v)$ we are using the van-Vleck Weisskopf profile which is most suitable for the microwave/terahertz domain [44]. 


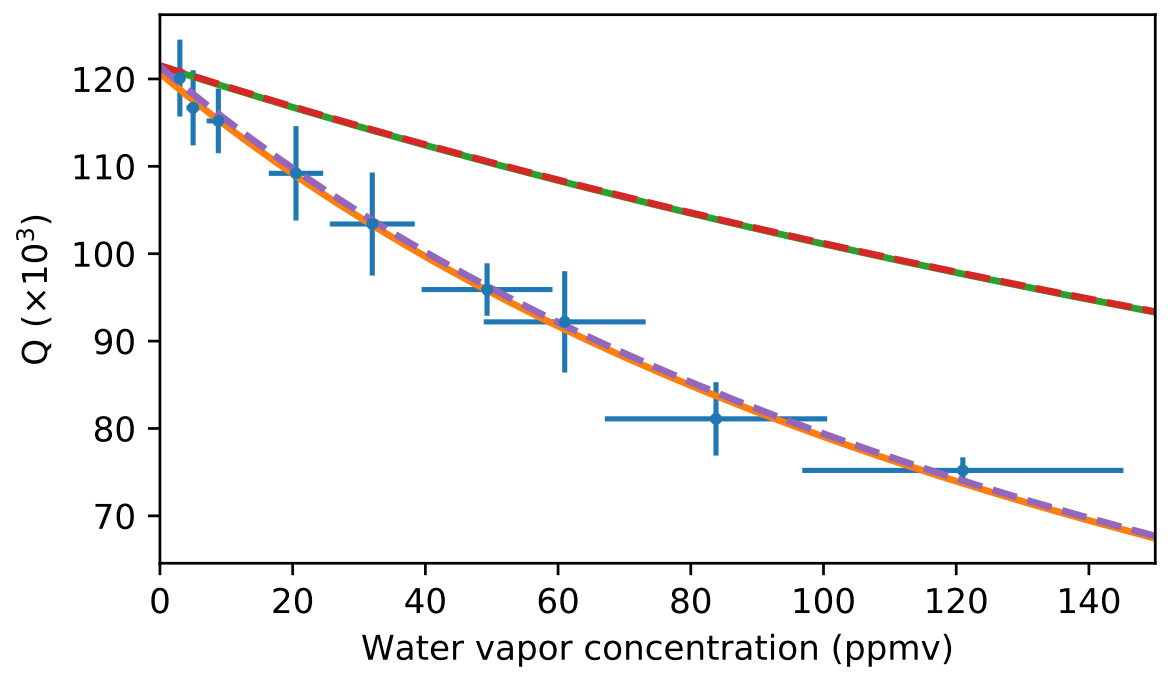

Figure 4. Measured Q-factors (blue dots with error-bars) as a function of water vapour concentration, with the corresponding fit (Equation (1), orange solid line). The calculated and simulated Q (ppmv) curves using the HITRAN database are shown with a green solid and red dashed lines, respectively. The simulated curve assuming a continuously growing water layer film on the disc is shown with the purple dashed line. The water layer is modelled as a Transition Boundary Condition with a uniform coverage of the disc, and an effective layer thickness. The dielectric function assumed for the liquid water layer is $\epsilon=4.8+i 3.2$ [45].

Interestingly, while the experimental data follows the trend predicted by Equation (1), the calculated $Q$ vs. ppmv dependence (combining Equations (1) and (2)) using the values from the HITRAN database (green solid curve) produces a curve that indicates lower absorption than the experimental results. However, the calculated and simulated (red dashed line) $\mathrm{Q}$ vs ppmv dependence are in excellent agreement, confirming the analytical approach described above. A possible explanation for this discrepancy could be the presence of a thin layer of liquid water adsorbed to the surface of the microresonator. The adsorbance of water is a ubiquitous process [46], and has been previously reported to reduce the ultrahigh Q-factor of microresonators in the optical domain $[47,48]$. Here, for example, at $80 \mathrm{ppmv}$ we get quantitative agreement with the experiments if we assume a uniform coverage of the disc with a water layer of about $3 \AA$ thickness, corresponding to about 1 mono-layer of water [46]. While this is a relatively thick coverage of the disc, at the given water vapour concentration, in comparison to reports in the literature, an increase in layer thickness from $1.5 \AA$ at 40 ppmv to $3 \AA$ at 80 ppmv would perfectly explain our results (see purple dashed curve in Figure 4). While there is undoubtedly water adsorbed to the $\mathrm{THz}$ disc microresonator, unfortunately we are not able to provide any further evidence about the thickness of the liquid water layer, as we have no other means to determine the thickness.

Nevertheless, the experimental results clearly show that the $\mathrm{THz}$ disc microresonator with sub-wavelength thickness can detect water vapour concentrations as low as 3 ppmv by interrogation of the absorption line at $0.5569 \mathrm{THz}$. Noteworthy, the sensitivity of the $\mathrm{THz}$ disc microresonator solely originates from the large evanescent field, and has not been enhanced using humidity absorbing materials like silica gel or surface activation [49,50]. Consequently, the reduction in Q-factor is immediate and shows no hysteresis as is common for many commercial hygrometers.

In general, due to the proportionality of the Q-factor to the inverse of the gas absorption (see Equation (1)), the microresonator shows the highest sensitivity at gas vapour concentrations leading to Q-factors close to the intrinsic Q-factor. Furthermore, the higher the intrinsic Q-factor, the steeper the drop-off in Q-factor with increasing gas concentration. Considering the experimental results presented in Figure 4, the best operation range for this resonator is estimated to be in the 
range from $0 \mathrm{ppmv}$ to $1000 \mathrm{ppmv}(0-4 \%$ relative humidity) water vapour concentration. In order to cover a wider range of water vapour concentrations, a combination of $\mathrm{THz}$ disc microresonators with various intrinsic Q-factors could be envisioned (with a lower/higher intrinsic Q-factor for higher/lower water vapour concentrations). Finally, the regularly spaced resonances over a broad frequency range (up to $200 \mathrm{GHz}$, see for example, Figure 1c, render the sub-wavelength thick $\mathrm{THz}$ disc microresonators ideal for the detection of gas mixtures (provided that the resonances coincide with specific gas absorption lines).

\section{Conclusions}

The presented results clearly demonstrate the sensing capabilities of the gas-phase $\mathrm{THz}$ spectrometer utilising the ultrahigh-Q THz disc microresonator. Minute water vapour concentrations in the ppm concentration level are detected without the need for a complex and bulky multi-pass absorption cell. In particular, the experimental data agrees very well with a calibration curve provided by a high-end commercial hygrometer. Additionally, perfect quantitative agreement is achieved by considering a few angstrom thick layer of liquid water adsorbed to the ultrahigh-Q THz disc microresonator. Ultimately, even higher sensitivities are within reach by further optimising the resonator design and utilising $\mathrm{THz}$ spectrometers with higher frequency resolutions, like a comb-locked frequency-domain spectrometer. The presented results are alluding to the possibility of compact and highly sensitive $\mathrm{THz}$ gas-phase spectrometer contributing to a wide range of applications.

Author Contributions: Data curation, D.W.V. and A.H.J.; Investigation, D.W.V., A.H.J. and R.L.; Methodology, D.W.V.; Project administration, D.W.V., R.L.; Software, D.W.V.; Supervision, D.W.V. and R.L.; Writing一original draft, D.W.V.; Writing-review \& editing, D.W.V., A.H.J. and R.L. All authors have read and agreed to the published version of the manuscript.

Funding: This research received no external funding.

Acknowledgments: The authors thank Harry L. Oudenhoven for his technical support in design and fabrication of the gas-cell, and acknowledge the support from the Dodd-Walls Centre for Photonic and Quantum Technologies.

Conflicts of Interest: The authors declare no conflict of interest.

\section{References}

1. Tonouchi, M. Cutting-edge terahertz technology. Nat. Photonics 2007, 1, 97-105. [CrossRef]

2. Siegel, P.H. Terahertz technology. IEEE Trans. Microw. Theory. Tech. 2002, 50, 910-928. [CrossRef]

3. Ferguson, B.; Zhang, X.C. Materials for terahertz science and technology. Nat. Mater. 2002, 1, $26-33$. [CrossRef]

4. Chan, W.L.; Deibel, J.; Mittleman, D.M. Imaging with terahertz radiation. Rep. Prog. Phys. 2007, 70, 1325. [CrossRef]

5. Pickwell, E.; Cole, B.E.; Fitzgerald, A.; Pepper, M.; Wallace, V. In vivo study of human skin using pulsed terahertz radiation. Phys. Med. Biol. 2004, 49, 1595. [CrossRef]

6. Fukunaga, K.; Ogawa, Y.; Hayashi, S.; Hosako, I. Terahertz spectroscopy for art conservation. IEICE Electron. Express 2007, 4, 258-263. [CrossRef]

7. Kemp, M.C.; Taday, P.; Cole, B.E.; Cluff, J.; Fitzgerald, A.J.; Tribe, W.R. Security applications of terahertz technology. Proc. SPIE 2003, 5070. [CrossRef]

8. Jepsen, P.U.; Cooke, D.G.; Koch, M. Terahertz spectroscopy and imaging-Modern techniques and applications. Laser Photonics Rev. 2011, 5, 124-166. [CrossRef]

9. Smith, R.M.; Arnold, M.A. Selectivity of terahertz gas-phase spectroscopy. Anal. Chem. 2015, 87, 10679-10683. [CrossRef]

10. Neumaier, P.; Schmalz, K.; Borngräber, J.; Kissinger, D.; Hübers, H.W. Terahertz gas-sensors: Gas-phase spectroscopy and multivariate analysis for medical and security applications. In Proceedings of the 2015 IEEE SENSORS, Busan, Korea, 1-4 November 2015.

11. Rothbart, N.; Holz, O.; Koczulla, R.; Schmalz, K.; Hübers, H.W. Analysis of human breath by millimeter-wave/terahertz spectroscopy. Sensors 2019, 19, 2719. [CrossRef] 
12. Hepp, C.; Lüttjohann, S.; Roggenbuck, A.; Deninger, A.; Nellen, S.; Göbel, T.; Jörger, M.; Harig, R. A cw-terahertz gas analysis system with ppm detection limits. In Proceedings of the 2016 41st International Conference on Infrared, Millimeter, and Terahertz Waves (IRMMW-THz), Copenhagen, Denmark, 25-30 September 2016; pp. 1-2.

13. Hindle, F.; Bocquet, R.; Pienkina, A.; Cuisset, A.; Mouret, G. Terahertz gas phase spectroscopy using a high-finesse Fabry-Pérot cavity. Optica 2019, 6, 1449-1454. [CrossRef]

14. Vahala, K.J. Optical microcavities. Nature 2003, 424, 839-846. [CrossRef] [PubMed]

15. Herr, T.; Brasch, V.; Jost, J.D.; Wang, C.Y.; Kondratiev, N.M.; Gorodetsky, M.L.; Kippenberg, T.J. Temporal solitons in optical microresonators. Nat. Photonics 2014, 8, 145-152. [CrossRef]

16. Spillane, S.; Kippenberg, T.; Vahala, K.; Goh, K.; Wilcut, E.; Kimble, H. Ultrahigh-Q toroidal microresonators for cavity quantum electrodynamics. Phys. Rev. A 2005, 71, 013817. [CrossRef]

17. Herr, T.; Hartinger, K.; Riemensberger, J.; Wang, C.; Gavartin, E.; Holzwarth, R.; Gorodetsky, M.; Kippenberg, T. Universal formation dynamics and noise of Kerr-frequency combs in microresonators. Nat. Photonics 2012, 6, 480-487. [CrossRef]

18. Del'Haye, P.; Schliesser, A.; Arcizet, O.; Wilken, T.; Holzwarth, R.; Kippenberg, T.J. Optical frequency comb generation from a monolithic microresonator. Nature 2007, 450, 1214-1217. [CrossRef] [PubMed]

19. Armani, D.; Kippenberg, T.; Spillane, S.; Vahala, K. Ultra-high-Q toroid microcavity on a chip. Nature 2003, 421, 925-928. [CrossRef]

20. Pfeifle, J.; Brasch, V.; Lauermann, M.; Yu, Y.; Wegner, D.; Herr, T.; Hartinger, K.; Schindler, P.; Li, J.; Hillerkuss, D.; et al. Coherent terabit communications with microresonator Kerr frequency combs. Nat. Photonics 2014, 8, 375-380. [CrossRef]

21. Xiao, S.; Khan, M.H.; Shen, H.; Qi, M. Multiple-channel silicon micro-resonator based filters for WDM applications. Opt. Express 2007, 15, 7489-7498. [CrossRef]

22. Shitikov, A.; Bilenko, I.; Kondratiev, N.; Lobanov, V.; Markosyan, A.; Gorodetsky, M. Billion Q-factor in silicon WGM resonators. Optica 2018, 5, 1525-1528. [CrossRef]

23. Mathai, C.; Jain, R.; Achanta, V.; Duttagupta, S.; Ghindani, D.; Joshi, N.; Pinto, R.; Prabhu, S. Sensing at terahertz frequency domain using a sapphire whispering gallery mode resonator. Opt. Lett. 2018, 43, 5383-5386. [CrossRef] [PubMed]

24. Vogt, D.W.; Leonhardt, R. Terahertz whispering gallery mode bubble resonator. Optica 2017, 4, 809-812. [CrossRef]

25. Vogt, D.W.; Jones, A.H.; Schwefel, H.G.; Leonhardt, R. Prism coupling of high-Q terahertz whispering-gallery-modes over two octaves from $0.2 \mathrm{THz}$ to $1.1 \mathrm{THz}$. Opt. Express 2018, 26, 31190-31198. [CrossRef] [PubMed]

26. Preu, S.; Schwefel, H.; Malzer, S.; Döhler, G.; Wang, L.; Hanson, M.; Zimmerman, J.; Gossard, A. Coupled whispering gallery mode resonators in the Terahertz frequency range. Opt. Express 2008, 16, 7336-7343. [CrossRef]

27. Vogt, D.W.; Leonhardt, R. Fano resonances in a high-Q terahertz whispering-gallery mode resonator coupled to a multi-mode waveguide. Opt. Lett. 2017, 42, 4359-4362. [CrossRef]

28. Xie, J.; Zhu, X.; Zang, X.; Cheng, Q.; Chen, L.; Zhu, Y. Terahertz integrated device: high-Q silicon dielectric resonators. Opt. Mater. Express 2018, 8, 50-58. [CrossRef]

29. Vogt, D.W.; Leonhardt, R. Ultra-high $\mathrm{Q}$ terahertz whispering-gallery modes in a silicon resonator. APL Photonics 2018, 3, 051702. [CrossRef]

30. Vogt, D.W.; Jones, A.H.; Schwefel, H.G.; Leonhardt, R. Anomalous blue-shift of terahertz whispering-gallery modes via dielectric and metallic tuning. Opt. Lett. 2019, 44, 1319-1322. [CrossRef]

31. Vogt, D.W.; Jones, A.H.; Leonhardt, R. Free-space coupling to symmetric high-Q terahertz whispering-gallery mode resonators. Opt. Lett. 2019, 44, 2220-2223. [CrossRef]

32. COMSOL A.B.; Stockholm, S. COMSOL Multiphysics ${ }^{\circledR}$ Software. Available online: www.comsol.com (accessed on 25 May 2020).

33. Gorodetsky, M.L.; Ilchenko, V.S. Optical microsphere resonators: optimal coupling to high-Q whispering-gallery modes. JOSA B 1999, 16, 147-154. [CrossRef]

34. Gordon, I.E.; Rothman, L.S.; Hill, C.; Kochanov, R.V.; Tan, Y.; Bernath, P.F.; Birk, M.; Boudon, V.; Campargue, A.; Chance, K.; et al. The HITRAN2016 molecular spectroscopic database. J. Quant. Spectrosc. Radiat. Transf. 2017, 203, 3-69. [CrossRef] 
35. Vogt, D.W.; Jones, A.H.; Haase, T.A.; Leonhardt, R. Sub-wavelength thick ultrahigh-Q terahertz disc microresonators. Photonics Res. 2020, accepted. [CrossRef]

36. Deninger, A.J.; Roggenbuck, A.; Schindler, S.; Preu, S. $2.75 \mathrm{THz}$ tuning with a triple-DFB laser system at $1550 \mathrm{~nm}$ and InGaAs photomixers. J. Infrared Millim. Terahertz Waves 2015, 36, 269-277. [CrossRef]

37. Vogt, D.W.; Erkintalo, M.; Leonhardt, R. Coherent Continuous Wave Terahertz Spectroscopy Using Hilbert Transform. J. Infrared Millim. Terahertz Waves 2019, 40, 524-534. [CrossRef]

38. Vogt, D.W.; Leonhardt, R. High resolution terahertz spectroscopy of a whispering gallery mode bubble resonator using Hilbert analysis. Opt. Express 2017, 25, 16860-16866. [CrossRef] [PubMed]

39. Lo, Y.H.; Leonhardt, R. Aspheric lenses for terahertz imaging. Opt. Express 2008, 16, 15991-15998. [CrossRef] [PubMed]

40. Foreman, M.R.; Swaim, J.D.; Vollmer, F. Whispering gallery mode sensors. Adv. Opt. Photonics 2015, 7, 168-240. [CrossRef] [PubMed]

41. Vogt, D.W.; Jones, A.H.; Leonhardt, R. Thermal tuning of silicon terahertz whispering-gallery mode resonators. Appl. Phys. Lett. 2018, 113, 011101. [CrossRef]

42. Wang, Z.; Dong, G.; Yuan, S.; Chen, L.; Wu, X.; Zhang, X. Voltage-actuated thermally tunable on-chip terahertz filters based on a whispering gallery mode resonator. Opt. Lett. 2019, 44, 4670-4673. [CrossRef]

43. Puppe, T.A.; Mayzlin, Y.; Robinson-Tait, J.; Wilk, R. Comb-locked Frequency-domain terahertz spectrometer. In Proceedings of the 2019 44th International Conference on Infrared, Millimeter, and Terahertz Waves (IRMMW-THz), Paris, France, 1-6 September 2019; p. 1.

44. Yang, Y.; Mandehgar, M.; Grischkowsky, D. Determination of the water vapor continuum absorption by THz-TDS and Molecular Response Theory. Opt. Express 2014, 22, 4388-4403. [CrossRef]

45. Ro/nne, C.; Thrane, L.; Åstrand, P.O.; Wallqvist, A.; Mikkelsen, K.V.; Keiding, S.R. Investigation of the temperature dependence of dielectric relaxation in liquid water by $\mathrm{THz}$ reflection spectroscopy and molecular dynamics simulation. J. Chem. Phys. 1997, 107, 5319-5331. [CrossRef]

46. Asay, D.B.; Kim, S.H. Evolution of the adsorbed water layer structure on silicon oxide at room temperature. J. Phys. Chem. B 2005, 109, 16760-16763. [CrossRef] [PubMed]

47. Ma, Q.; Huang, L.; Guo, Z.; Rossmann, T. Spectral shift response of optical whispering-gallery modes due to water vapor adsorption and desorption. Meas. Sci. Technol. 2010, 21, 115206. [CrossRef]

48. Vernooy, D.; Ilchenko, V.S.; Mabuchi, H.; Streed, E.; Kimble, H. High-Q measurements of fused-silica microspheres in the near infrared. Opt. Lett. 1998, 23, 247-249. [CrossRef]

49. Su, J. Label-free biological and chemical sensing using whispering gallery mode optical resonators: Past, present, and future. Sensors 2017, 17, 540. [CrossRef]

50. Mallik, A.K.; Farrell, G.; Liu, D.; Kavungal, V.; Wu, Q.; Semenova, Y. Silica gel coated spherical micro resonator for ultra-high sensitivity detection of ammonia gas concentration in air. Sci. Rep. 2018, 8, 1620. [CrossRef]

(C) 2020 by the authors. Licensee MDPI, Basel, Switzerland. This article is an open access article distributed under the terms and conditions of the Creative Commons Attribution (CC BY) license (http:/ / creativecommons.org/licenses/by/4.0/). 